\title{
Measurement of the Tidal Dissipation in Multiple Stars
}

\author{
Andrei Tokovinin \\ Cerro Tololo Inter-American Observatory, Casilla 603 La Serena, Chile \\ e-mail: atokovinin@ctio.noao.edu
}

\begin{abstract}
Considerable effort has been spent to determine the period of tidal circularization in close binaries as a function of age, in order to constrain the tidal dissipation theory. A new, direct method of measuring the tidal dissipation by precise timings of periastron passages in eccentric binaries undergoing circularization is proposed. Such binaries with components just leaving the Main Sequence can be found as inner systems in multiple stars. Three examples are given.
\end{abstract}

\section{Introduction}

Tidal interaction in close binary stars is important in many respects. It provides a dissipative mechanism of orbit circularization, converting eccentric binaries with long periods into short-period systems with circular orbits. This orbital circularization is accompanied by the synchronization of component's axial rotation with the orbit. Both processes can act when stars are just forming, at the pre-Main Sequence (PMS) stage, on the Main Sequence (MS), or in systems with evolved components. In all cases the knowledge of the tidal forces is needed to understand the physics and evolution of binary systems, including some special important cases such as X-ray binaries or pulsars. Here I propose a new method that can potentially lead to a direct measurement of the dissipation.

Traditionally, the tidal dissipation in Main Sequence (MS) stars is constrained from the circularization periods $P_{\text {circ }}$ in open clusters. Binaries with $P<P_{\text {circ }} \approx 10^{\text {d }}$ have circular orbits while orbits with longer periods are eccentric. The $P_{\text {circ }}$ is only an indirect measure of tidal circularization, being dependent on unknown initial conditions and evolutionary history. Its determination is affected by the small number of short-period binaries in each cluster. Meibom \& Mathieu (2005) reach a conclusion that current theories of tidal interaction explain circularization and synchronization qualitatively, but not quantitatively.

The circularization with constant angular momentum follows the track $P\left(1-e^{2}\right)^{3 / 2}=$ const, i.e. is accompanied by the shortening of the orbital period. The period can be determined with a much better precision than eccentricity. A detection of period changes will lead to the measurement of the tidal dissipation rate. In interpreting these variations, we will have to account for the period changes caused by other effects such as by the exchange of the angular momentum with components, dynamical tides, etc.

A measurable period change caused by tidal dissipation happens in binaries with a short circularization time-scale. Evidently, such binaries are rare. They can be found among short-period PMS binaries with eccentric (still circularizing) orbits. Another option is to study inner sub-systems in multiple stars with sub-giant components, as explained below. 


\section{Origin and destiny of very eccentric binaries}

The origin of very high eccentricities is naturally explained by Kozai cycles - strong eccentricity modulation in triple systems with high mutual orbit inclinations (Eggleton 2006). These cycles are softened by the relativistic apsidal motion (AM): a short-period sub-system cannot acquire very large eccentricities by the Kozai mechanism if its tertiary companion is too distant. The Kozai cycles are also perturbed by the tidal friction, so that eventually (i) the inner binary becomes locked into the high-e state of the cycle, and then (ii) the eccentricity decreases on the frictional timescale, with angular momentum being roughly constant, till circularity is reached. The orbit evolves on a path like the dash-dotted line of Fig. 1. The product of such evolution will be a spectroscopic binary with giant components and near-circular orbit. There are many such systems among known multiple stars (Tokovinin 1997). The existence of highly eccentric sub-systems in multiples is not a rare event but rather a natural outcome of the dynamical evolution.

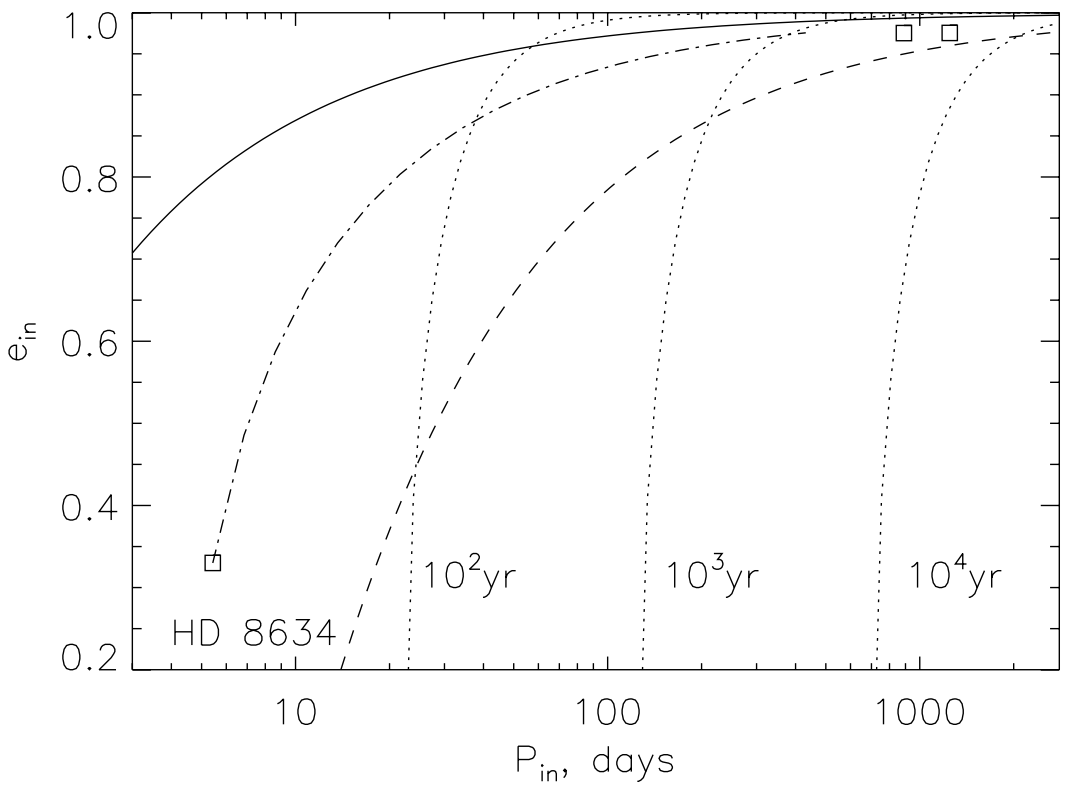

Figure 1. Eccentricity of inner sub-systems in triple stars vs. their period. The full line is the $P, e$ relation when the two components just touch each other at periastron for a sum of radii equal $3.6 R_{\odot}$ (like in $41 \mathrm{Dra}$ ). The dashed line is the $P, e$ locus for orbits which have the same periastron distance as a 10-day circular orbit, $P(1-e)^{3 / 2}=10^{\mathrm{d}}$. The dotted lines are the maximum eccentricities of Kozai cycles softened by the relativistic AM, for 3 representative tertiary periods $P_{\text {out }}^{*}$. The squares denote three systems discussed in the text. The dash-dot line is the line along which HD 8634 must have evolved at constant angular momentum but decreasing $e$ to reach its present position.

The squares in Fig. 1 are the real systems illustrating this scenario. Gliese 586A (Duquennoy et al. 1992) held for a while the world record of eccentricity, $e=0.9752$. It is a pair of unevolved low-mass dwarfs accompanied by a physical component B and an even more distant common proper motion companion C at 20.2' (24000 AU). The circularization of this system was studied by Goldman \& Mazeh (1994). Rapid circularization is not expected in this system. 41 Dra (Tokovinin et al. 2003) has an even larger eccentricity $e=0.9754$ and forms a physical quadruple with a $10.5^{\mathrm{d}}$ spectroscopic binary 
40 Dra. These F-type stars are moderately evolved, the age is estimated as 2.5 Gyr by isochrone fitting. As the component's radius increases, so does the tidal dissipation. Thus 41 Dra may have already started its phase of rapid circularization.

HD $8634=$ HR 407 is a binary with $P=5.4^{\mathrm{d}}<P_{\text {circ }}$ and $e=0.33$. The orbit was computed by Wright \& Pugh (1954) and later re-determined by Mayor \& Mazeh (1987) in search of precession caused by a tertiary companion. No precession was found, yet a faint tertiary at $1.5^{\prime \prime}$ has been discovered with adaptive optics by Tokovinin et al. (2006). The primary F5III component is slightly evolved. It was a late-A type star on the MS. It is likely that HD 8634 is now undergoing rapid circularization, evolving from a high-eccentricity state.

\section{Can we observe the tidal circularization?}

Uncertainties in the tidal theory do not permit reliable estimation of the circularization time scale for very eccentric binaries (Goldman \& Mazeh 1994), hence a direct measurement of the orbital evolution in a system like 41 Dra will be of great value. The radial velocity at periastron changes by $3 \mathrm{~km} \mathrm{~s}^{-1}$ per hour. Presently we measure the periastron time (and period) to $0.1 \mathrm{~h}$ with radial velocities accurate to $0.3 \mathrm{~km} \mathrm{~s}^{-1}$. With a conservative 10-fold increase in precision we hope to bring the timing error down to $0.01 \mathrm{~h}$. This gives a measurable effect $\mathrm{d} P / P=3.34 \times 10^{-7}$ (time scale of $3 \mathrm{Myr}$ ). The next periastron passage will occur on April 1, 2008.

The case of HD 8634 is tantalizing. Application of Eq. 2 of Verbunt \& Phinney (1995) to a similar system with a convective primary gives the circularization time of $\sim 4$ Myr. If a new, precise periastron timing is made now, the period variation on a time scale of $10 \mathrm{Myr}$ or shorter is detectable. Complications such as motion around the tertiary and spin-orbit interaction must be addressed when interpreting future timings.

\section{Conclusions}

It is shown that binary stars undergoing presently rapid tidal orbital evolution are formed naturally within multiple systems. Period changes in such systems can be detected and will provide a direct measurement of the tidal dissipation. Monitoring a sample of (otherwise uninteresting) spectroscopic binaries with precise RV techniques over a long period of time will eventually lead to the successful detection of orbital tidal evolution.

\section{References}

Duquenoy, A., Mayor, M., Andersen, J. et al. 1992, A\&GA 254, L13

Eggleton P. 2006, Evolutionary Processes in Binary and Multiple Stars. (Cambridge, UK: Cambridge Univ. Press)

Goldman, I. \& Mazeh, T. 1994, ApJ 429, 362

Mayor, M. \& Mazeh, T. 1987, A\& A 171, 157

Meibom, S. \& Mathieu, M. 2005, AJ 620, 970

Tokovinin, A. 1997, A\&AS 124, 75

Tokovinin, A., Balega, Y.Y., Pluzhnik, E.A. et al. 2003, A\&A 409, 245

Tokovinin, A., Thomas, S., Sterzik, M., \& Udry, S. 2006, A\&A 450, 681

Verbunt, F. \& Phinney, E.S. 1995, A\&\&A 296, 709

Wright, K.O. \& Pugh, R.E. 1954, Publ. DAO 9, 407 